\title{
INDOOR HEADING MEASUREMENT SYSTEM
}

\author{
Marius Malcius \\ Department of Research and \\ Development \\ AB "Prospero polis", Lithuania \\ m.malcius@korodur.lt
}

\author{
Darius Munčys \\ Department of Research and \\ Development \\ AB "Prospero polis", Lithuania \\ d.muncys@korodur.lt
}

\author{
Šarūnas Atkočiūnas \\ sarunas.atkociunas@gmail.com
}

\begin{abstract}
This paper analyses iGPS as stand alone system for vehicle heading measurement and presents iGPS and gyro fusion algorithm based on Kalman filter. This solution has advantage of reduced heading measurement delay, higher measurement update ratio and also increased resistance to iGPS outage, which makes it more suitable for indoor and outdoor precise navigation.
\end{abstract}

\section{KEYWORDS}

Heading measurement, Kalman filter, indoor GPS

\section{INTRODUCTION}

There were a lot of more or less successful attempts during the last years to develop autonomous or partially autonomous vehicles, that can move in given trajectory, while doing assigned jobs. Knowledge of their precise orientation is critical for control of these types of vehicles (robots). Efficiency and usability of such vehicles is directly dependent on orientation and position measurement accuracy. Indoor environments are especially demanding on position and orientation measurement systems. There is no way to use widespread GPS navigation system in indoor environments (i.e. warehouse). Orientation measurement system based on magnetometers is difficult to use because of hard iron errors. Therefore the number of commercially available devices for robot orientation measurements with acceptible performance in indoor environments is reduced.
In this paper both static and dynamic heading measurements performed using commercially available indoor GPS system as well as its fusion with angular rate gyro is discussed.

\section{IGPS}

iGPS is laser based 3D position measurement system which consists of laser transmitters, sensors, Receiver Hub (RH) (Figure 1) and main server for calculating sensor position from data supplied by $\mathrm{RH}$ through wired or wireless connection.

Manufacturer of iGPS declares $0.25 \mathrm{~mm}$ uncertainty of static measurements in 3D space (while averaging 100 measurements), maximum measurement frequency of $40 \mathrm{~Hz}$ and a maximum distance between one transmitter and sensor of $40 \mathrm{~m}$ [1]. 


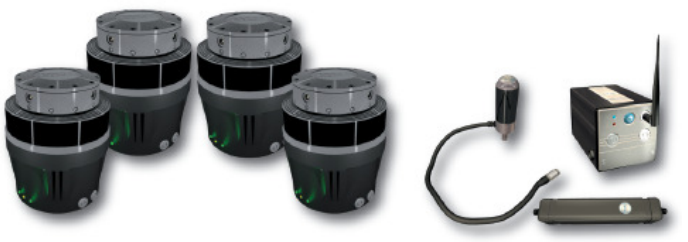

Figure 1. Hardware parts of iGPS system

\section{WORKING PRINCIPLES}

Reference points for the usual Global Positioning System (GPS) are satellites. Pseudoranges between satellites and receiver are calculated and used in further calculations to get receiver position which in turn can be transformed in any desired coordinate system. Transmitters act as reference points for iGPS. They are distributed in working area and used to calculate position of the sensor.

Each transmitter emits two fan-shaped beams which are approximately $90^{\circ}$ apart horizontally when viewed from above the transmitter (Figure 2). The beams fan approximately $+/-30^{\circ}$ from the horizontal plane and each beam is also canted approximately $30^{\circ}$ from the vertical axis. A third optical signal is emitted from the transmitter at the start of every other rotation. This broad-area strobe serves as a timing signal for the receivers, and is emitted $360^{\circ}$ around the transmitter. Sensor detects both laser beams and strobe hit times. Time between strobe and first laser is used to calculate angle $\mathrm{Az}$ and the one between laser hits produces angle El. Each transmitter rotates at slightly different speed which enables differentiation between signals from different transmitters.

With two transmitters present we get four angles. Given that positions, orientations of transmitters and angle from each transmitter to the sensor are known, it is possible to use triangulation to calculate $3 \mathrm{D}$ position of the sensor itself [2].

Sensors are connected to RH where initial signal processing takes place. Processed data is sent by 2.4 $\mathrm{GHz}$ radio frequency or through RS232 interface to PC, where appropriate software that calculates sensor positions is running (Figure 3).

\section{Advantages of iGPS:}

- System can work in both indoor and outdoor environments.

- $\quad 0.25 \mathrm{~mm}$ uncertainty of static position measurements.

- Maximum measuring frequency of $40 \mathrm{~Hz}$.

- Easy set-up in new working environment.

- Scalability. There is no limit for number of positioned sensors in working area.

Disadvantages of iGPS:

- Sensor needs direct line of sight to at least 2 transmitters for successful positioning.

- Reflective surfaces such as wet floor or windows create multipath situations that have negative impact on system's performance.

- Position calculation subsystem requires. NET framework version 2.0.
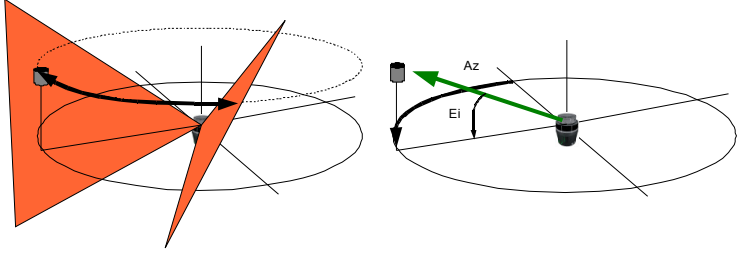

Figure 2. Working principles of transmitter

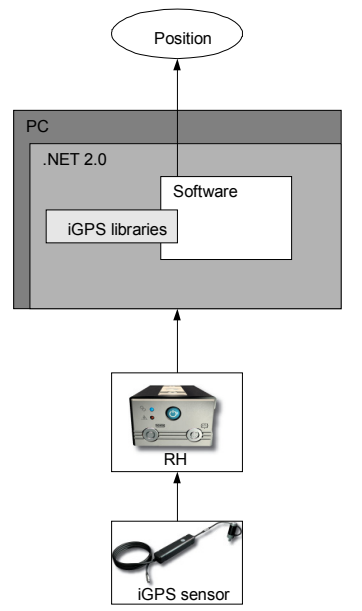

Figure 3. iGPS data flow diagram 


\section{HEADING CALCULATION USING POSITIONS OF TWO SENSORS}

While iGPS system can track 3D position of a sensor, there are many applications where 2D position measurements are sufficient for vehicle control purposes. Therefore in this paper we will only use planar position measurements.

When 2D positions of two specific points on a vehicle are known, it's possible to calculate its heading (heading is defined as yaw angle from OX axis of iGPS coordinate system) using following algorithm (Figure 4):

Heading $\theta$ is considered to be a direction dependent on the direction of vector $\overrightarrow{S_{1} S_{2}}$ and differing from it by certain offset. In this particular case $\pi / 2$ was chosen as an offset. Function atan2 was used to find the direction of vector $\overrightarrow{S_{1} S_{2}}$ and offset was added to it.

$\theta=\operatorname{atan} 2\left(\mathrm{y}_{2}-\mathrm{y}_{1}, \mathrm{x}_{2}-\mathrm{x}_{1}\right)+\pi / 2$

\section{EXPERIMENT ENVIRONMENT}

While testing iGPS as a dynamic heading measurement tool the platform was chosen where iGPS sensors were placed $41 \mathrm{~cm}$ apart from each other. The center of the platform is attached to an angle encoder for reference heading data. When the platform is turning around its axis, the iGPS sensors are moving in a circle whose plane is parallel to the xy plane of the iGPS coordinate system. The heading of the platform is calculated by measuring positions of the sensors and using equation (1).

Three iGPS transmitters were used for the experiment. They were distributed in $5 \times 5 \mathrm{~m}$ area. RS232 interface was used to connect RH module to embedded PC that was used to calculate heading of the platform (Figure 5).

\section{RESULTS OF IGPS HEADING MEASUREMENT}

Static and dynamic heading measurements were tested using iGPS. During measurement test of constant heading, the platform was set in zero heading position and was not moved.

The results of the experiment are shown in Figure 6

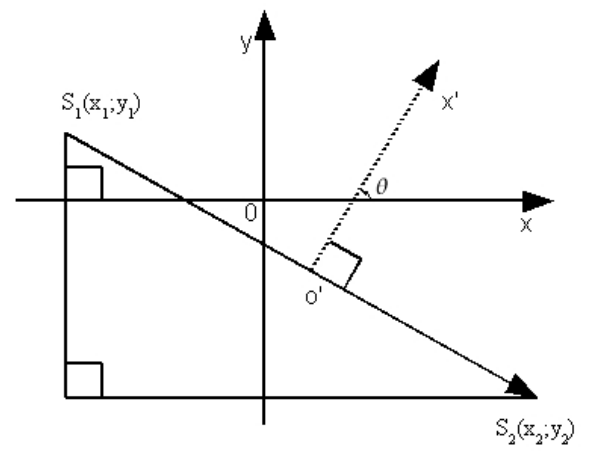

Figure 4. Heading calculation

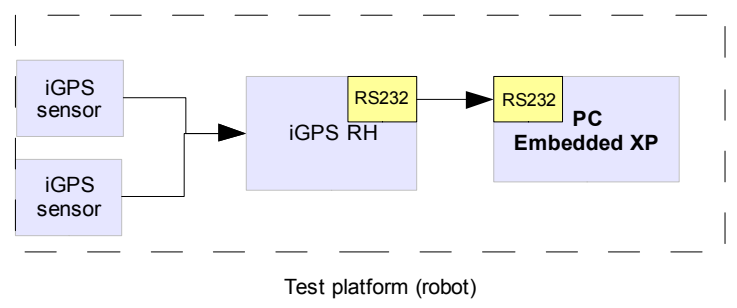

Figure 5. System architecture

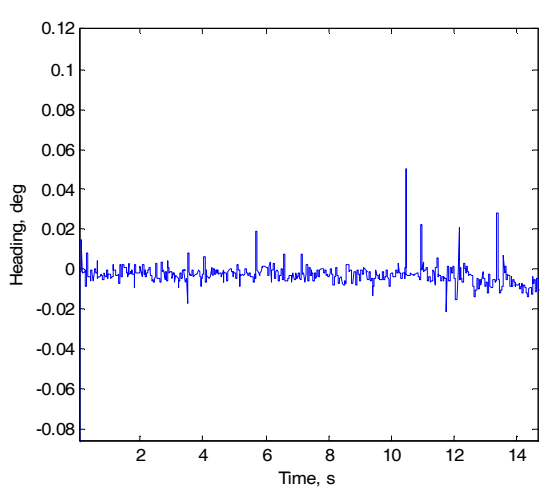

Figure 6. Static heading measurement

Heading data standard deviation is 0.0048 deg.

Resolution and accuracy of the static heading measurement can be further improved by increasing the distance between sensors.

During dynamic heading measurement test, the platform was turned around its axis in both directions. The results are displayed in Figure 7. 


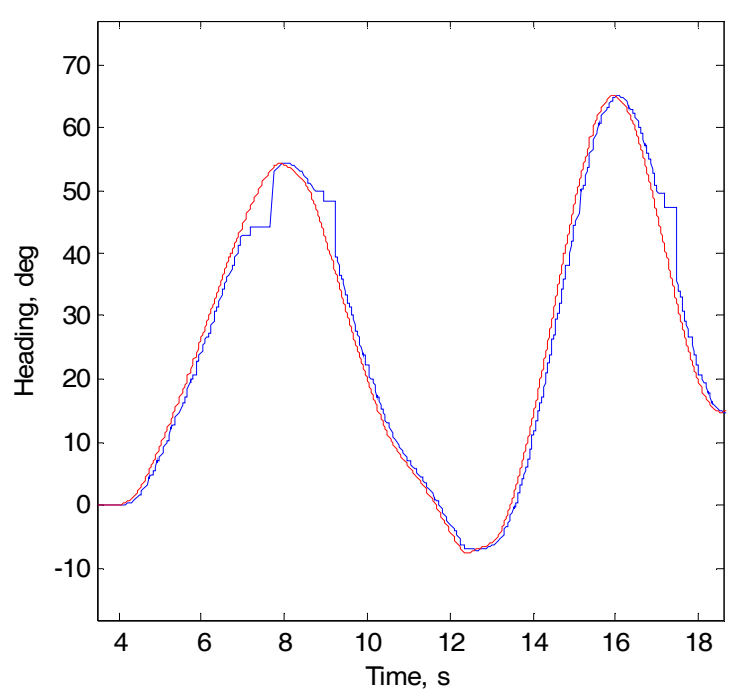

Figure 7. Test results. Red line - true heading, blue heading measured with iGPS

As seen in Figure 7 there is about $100 \mathrm{~ms}$ delay in iGPS heading measurements. One of heading error components directly depends from the rate in which heading is changing and can be expressed by following equation:

$$
\delta \theta=\int_{t-\text { delay }}^{t} \omega d t
$$

where $\omega$ is heading change rate.

The average measurements frequency during the experiment was $37 \mathrm{~Hz}$. As displayed in Figure 8, time between measurements usually varies between 0.02 and $0.08 \mathrm{~s}$ with occasional spikes up to $0.18 \mathrm{~s}$. During the whole duration of the experiment the longest observed time between measurements was $0.5 \mathrm{~s}$. Such behavior can be explained by multipath cases (sensor receives both real and reflected signals so they both are ignored) and occasionally blocked line of sight between sensor and transmitter.

\section{SENSOR FUSION}

Sensor fusion is commonly used to merge several signals from different systems, extracting a better, more reliable result. This has shown to be very successful for solving navigation problems when using sensors with different strengths like GPS and INS $[3,4,5]$.

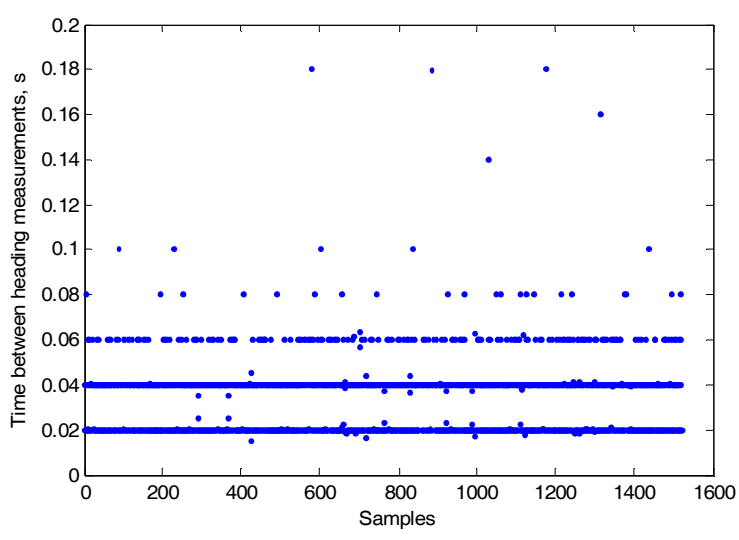

Figure 8. Measurement intervals

In order to improve yaw angle measurement dynamic parameters such as latency, measurement update rate and also to minimize time intervals when heading measurement is unavailable because of multipath or iGPS transmitters' blockage it was decided to use Kalman filter for iGPS and gyro (which was mounted on test platform) data fusion. iGPS is an external or absolute sensor, thus the errors in the data it provides are bounded. However in difficult environments such as construction site, multipath or transmitter blockage is common which leads to heading measurement interruptions. The primary advantage of rate gyro sensor is that angular rotation data is provided at high update rates (100 Hz or more). Main problem with gyroscope is an error caused by bias in sensor readings which accumulates with time. Fusion of these two sensors should improve overall heading measurement performance.

\section{KALMAN FILTER}

A linear discrete system observed by measurements where both process and measurement are influenced by additive Gaussian noise can be put in a state space form as follows:

$$
\begin{aligned}
& x_{k+1}=A_{k} x_{k}+B_{k} u_{k}+\omega_{k} \\
& z_{k}=C_{k} x_{k}+v_{k}(4)
\end{aligned}
$$

where: $\omega_{k} \sim N\left(0, Q_{k}\right)$ and $v_{k} \sim N\left(0, R_{k}\right)$. 
We assume that two noise sources $\omega_{\mathrm{k}}$ and $\mathrm{v}_{\mathrm{k}}$ are independent $E\left(\omega_{i}, v_{j}^{T}\right)=0$. To minimize variance of state estimation error Klaman filter is used. A proof along with derivation of Kalman filter equations can be found in [7]. The summary of equations is listed below:

$$
\begin{aligned}
& \hat{x}_{k+1}=A_{k} \hat{x}_{k}(+)+B_{k} u_{k} \\
& P_{k+1}=A_{k} P_{k}(+) A_{k}^{T}+Q_{k} \\
& K_{k}=P_{k} C_{k}^{T}\left[C_{k} P_{k} C_{k}^{T}+R_{k}\right]^{-1} \\
& \hat{x}_{k}(+)=\hat{x}_{k}+K_{k}\left[z_{k}-C_{k} x_{k}\right] \\
& P_{k}(+)=\left[I-K_{k} C_{k}\right] P_{k}
\end{aligned}
$$

\section{ERROR MODEL}

Basic attitude error model can be expressed as follows [8]:

$$
\delta \dot{\psi}=-C_{b}^{n} \delta \omega_{b}
$$

where $\delta \dot{\psi}$ and $\delta \omega_{b}$ are orientation errors in navigation frame and angular velocities errors in body frame respectively. $C_{b}^{n}$ is transformation matrix between body and navigation frames.

If object is moving only in the plane parallel to XY plane of navigation frame and both roll and pitch rates are zero then equation (10) can be simplified:

$$
\delta \dot{\theta}=-\delta \omega_{z b}
$$

where $\delta \dot{\theta}$ is heading error and $\delta \omega_{z b}$ is yaw angular velocity error.

Angular velocity error is a sum of several individual errors such as: bias, scale factor, misalignment and nonlinearity. All of these errors can be placed in state model to be estimated. For the sake of simplicity and because biases are dominating in lowgrade gyros, yaw angle rate error is modeled as constant and heading error model remains as represented in (11).
To estimate gyro bias complementary Kalman filter was applied to error model described by equation (11).

\section{RESULTS OF HEADING MEASUREMENT USING SENSOR FUSION}

Main purposes of using sensor fusion were to reduce heading measurement delay, vulnerability to iGPS outage and to increase measurement update rate. Using very simple heading error model (11) along with complementary Kalman filter, provide us with results shown in Figure 9.

By using sensor fusion algorithm described earlier heading measurement latency was reduced from 100 $\mathrm{ms}$ to $10 \mathrm{~ms}$ as shown in Figure 10. Measurement update rate was increased from $37 \mathrm{~Hz}$ on average to exactly $100 \mathrm{~Hz}$ (gyro sampling rate).

Measurement error of dynamically changing heading is several times smaller when using sensor fusion algorithm compared to stand alone iGPS (Figure 11) due to reduced latency. Heading error peaks coincide with highest yaw angle rates.

The results of the experiments are displayed in Table 1 . They may vary depending on yaw angle rate.

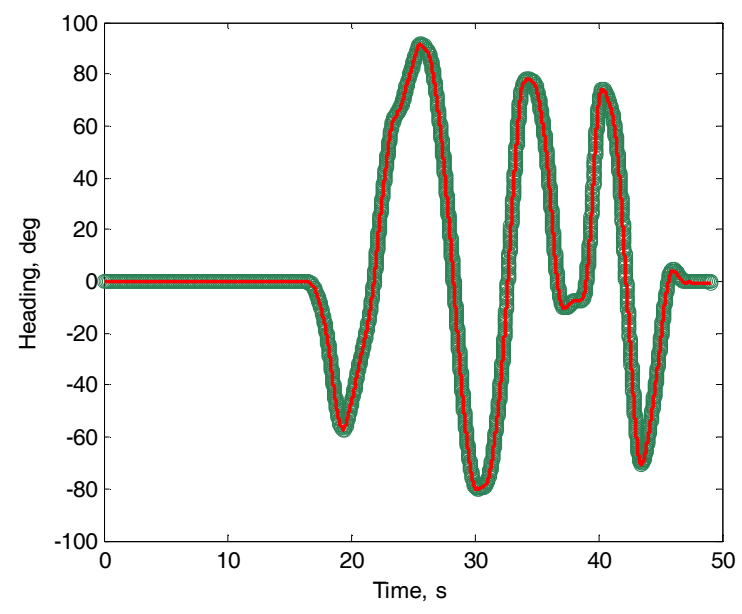

Figure 9. Actual heading - red line, iGPS and gyro fusion result - green line 


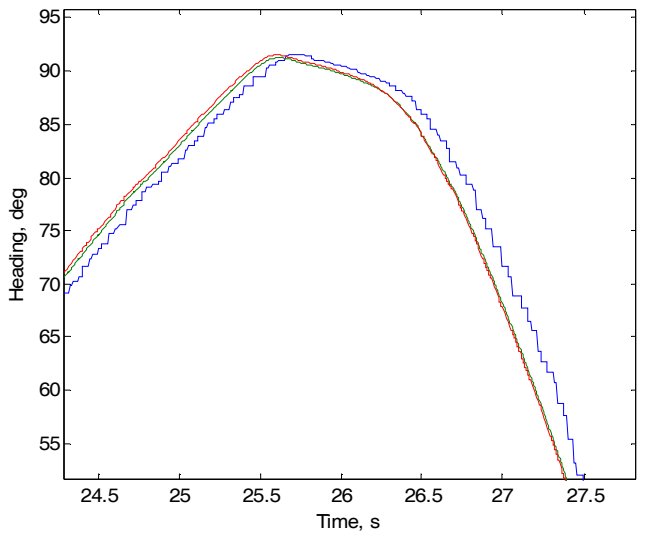

Figure 10. Actual angle - red line, stand alone iGPS heading - blue line, iGPS and gyro fusion result - green line

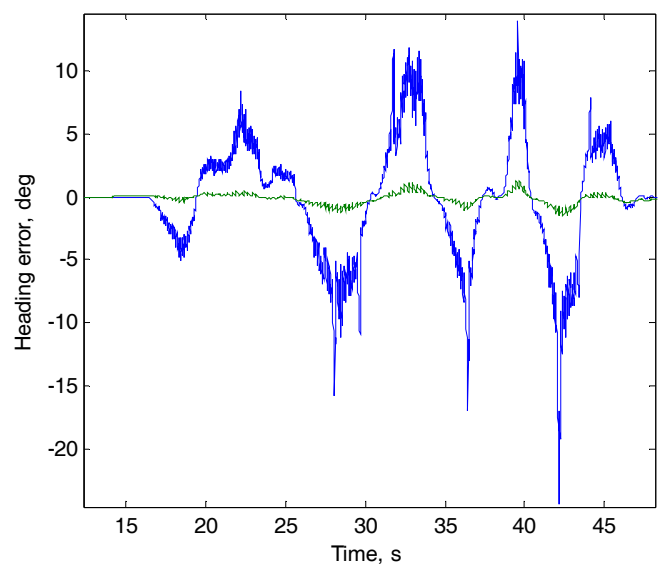

Figure 11. Stand alone iGPS heading error - blue line, iGPS and gyro fusion error - green line

Table 1. Test results

\begin{tabular}{|c|c|c|}
\hline Parameters & iGPS alone & $\begin{array}{c}\text { iGPS fused with } \\
\text { gyro }\end{array}$ \\
\hline Error std & $3.9212 \mathrm{deg}$ & $0.369 \mathrm{deg}$ \\
\hline Max error & $24.33 \mathrm{deg}$ & $1.5 \mathrm{deg}$ \\
\hline
\end{tabular}

\section{CONCLUSIONS}

Capabilities of iGPS as a heading measurement system were shown in this paper. Main disadvantage of using iGPS to measure heading is its measurement delay caused by system operation principles. Hence real time measurement of rapidly changing heading has error component proportional to the heading rate. Measurement delay was reduced from 100 to $10 \mathrm{~ms}$ by using Kalman filter for sensor fusion, which led to reduced heading measurement error component dependent on heading change rate. Measurement of dynamically changing heading could be further improved by using more precise angular velocity error model.

\section{REFERENCES}

[1] ArcSecond. (2002) "WorkSpace Advenced Metrology Edition User's Guide".

[2] ArcSecond. (2002) "Indoor GPS Technology For Metrology".

[3] Grewal, S.; Weill, R.; Andrews, P. (2001) "Global positioning system, inertial navigation, and integratio". John Willey \& Sons, ISBN-0-471-20071-9.

[4] Jekeli, C. (2001) "Inertial navigation systems with Geodetic Applications". - Berlin; New York: de Gruyter, ISBN 2-11-015903-1.

[5] Ronnback, S. (2000) "Development of a INS/GPS navigation loop for an UAV". 2000:081 ISSN: 14021617 ISRN: LTU-EX -00/081-SE.

[6] Linsong, G., Young, H. (2002) "Real-Time Tractor Position Estimation System Using a Kalman Filter". IEEE Article ID: 1002-6819(2002)-0096-06.

[7] Brookner, E. (1998) "Tracking and Kalman Filtering Made Easy". John Wiley \& Sons, Inc. ISBNs: 0-47118407-1 (Hardback); 0-471-22419-7 (Electronic)

[8] Sukkarieh, S., Nebot, E.M. and Durrant-Whyte, H.F. (1999) "A high integrity IMU GPS navigation loop for autonomous land vehicles applications". IEEE Trans. Robot. Autom. v15 i3. 572-578. 